\title{
Some Effects of Nystatin on the Growth of Four Aspergillus Species
}

\author{
BY VALERIE C. STANLEY* AND MARY P. ENGLISH \\ Mycology Laboratory, Bristol Royal Hospital, General Hospital Branch, Bristol 1
}

(Received 25 January 1965)

\begin{abstract}
SUMMARY
At subinhibitory concentrations nystatin exerted its main effect on four species of Aspergillus in the lag phase of growth. Subsequent growth and sporulation appeared to be normal, indicating the lack of permanent damage to survivors. Starting with an inoculum of spores the increased lag period was caused by the inhibition of swelling and germination; starting with an inoculum of mycelium, by the temporary cessation of hyphal elongation. At low concentrations of nystatin the lag period increased in proportion to the increase in initial nystatin concentration. Under these conditions the length of the lag was limited by the natural breakdown of the antibiotic in the medium rather than by any metabolic activity or adaptive response of the fungi. However, the lag period increased sharply, as the inhibitory nystatin concentration for each species was approached, to values greater than can be explained solely by the increased concentration of antibiotic. This was not due to the time taken for growth by any especially resistant spores but to the greatly decreased viability and slow outgrowth of normal survivors. This loss in viability was due to factors other than $\mathbf{K}^{+}$leakage. Plasmolysis of germinated spores and mycelium occurred in some Aspergillus species at high concentrations of nystatin. The action of nystatin was relatively slow; no significant loss of viability or lengthening of the lag phase was noted until after exposure to the antibiotic for at least one hour, even at very high concentrations. Since the fungicidal effect on Aspergillus terreus and $\boldsymbol{A}$. fumigatus was appreciably slower than on $A$. niger and $A$. flavus, it is possible that differences in the rate of absorption of nystatin in part determine sensitivity to nystatin. Spore germination took place at higher nystatin concentrations than did hyphal growth. The lethal dose for spores, which was three to four times higher than the fungistatic value, was also higher than for the mycelium.
\end{abstract}

\section{INTRODUCTION}

Nystatin, a polyene antifungal antibiotic obtained from Streptomyces noursei, is absorbed in significant amounts only by micro-organisms which are sensitive to it, that is, by certain yeasts, algae and filamentous fungi (Lampen, Morgan, Slocum \& Arnow, 1959; Lampen \& Arnow, 1961), their growth and utilization of various substrates being inhibited. These effects have been studied, in the main, in yeasts, relatively little work having been done with filamentous fungi. According to Lampen, Arnow, Borrowska \& Laskin (1962), $90 \%$ of the absorbed nystatin is bound by the yeast cell membrane. The membrane is damaged in the binding process,

\footnotetext{
* Present address : Bacteriology Department, Queen Charlotte's Maternity Hospital, Goldhawk
} Road, London, W. 6. 
which leads to leakage of cytoplasmic constituents, inhibition of glucose utilization and eventual death. In the filamentous fungus, Neurospora crassa, the loss of cytoplasmic constituents and lysis of the protoplasts by the action of nystatin have been demonstrated by Kinsky $(1961,1962)$, indicating again that the primary action of the antibiotic is due to a direct action on the membrane leading to an alteration of permeability.

During an examination of the effectiveness of nystatin as an antifungal antibiotic for the treatment of otomycosis (a fungal disease of the external auditory canal and of postoperative aural cavities) studies have been made of the action of nystatin on the growth of various Aspergillus species. Experiments were made to determine the cause of the delay in growth which occurs at subinhibitory nystatin concentrations and attempts made to discover whether this delay was due to the breakdown of nystatin to a concentration at which growth can occur, to the reversal of damage caused by nystatin, to adaptation by the fungi, or to a combination of these factors.

\section{METHODS}

The following fungi were used: one isolate each of Aspergillus flavus, A. terreus, $A$. niger and $A$. fumigatus all obtained from cases of otomycosis; a laboratory isolate of $A$. fumigatus ( $A$. fumigatus $\mathrm{NyR}$ ) with increased resistance to nystatin; a baking strain of Saccharomyces cerevisiae.

Stock cultures were grown for $48 \mathrm{hr}$ at $37^{\circ}$ on glucose + peptone agar (GP agar) slopes ( $4 \%$ glucose, $1 \%$ peptone, $2 \%$ agar, $\mathrm{pH} 5 \cdot 2)$, and then maintained at room temperature.

Spore suspensions were prepared by rubbing a $48 \mathrm{hr}$ slope culture on GP agar with a solution of $0.05 \%(v / v)$ Tween 80 in distilled water. Spore counts were made in a haemocytometer and the suspension adjusted to the required strength by adding more Tween 80 solution. A spore suspension of $6 \times 10^{7}$ spores $/ \mathrm{ml}$. (standard spore suspension) was used in all experiments unless stated otherwise.

In all experiments cultures were incubated at $37^{\circ}$.

Suspensions of germinated spores were obtained by mixing $2 \mathrm{ml}$. of a standard spore suspension with $2 \mathrm{ml}$. of glucose + peptone broth (GP broth) and incubating until microscopic examination showed that the majority of spores had germinated. For the production of mycelial balls, $0.1 \mathrm{ml}$. of a standard spore suspension was inoculated into $50 \mathrm{ml}$. flasks containing $20 \mathrm{ml}$. of GP broth. The flasks were then incubated on a reciprocating shaker for $24 \mathrm{hr}$ and the mycelial balls produced were harvested, washed and resuspended in fresh medium.

Thin seeded agar dises for radial growth experiments were prepared by inoculating $0.1 \mathrm{ml}$. of a standard spore suspension into $10 \mathrm{ml}$. of molten GP agar, cooled to $45^{\circ}$. The tubes were well mixed and plates poured. Dises of $0.9 \mathrm{~cm}$. diameter were cut out with a cork borer. When similar discs containing mycelium with the minimum degree of sporulation were required, the seeded agar was covered with cellulose film and the plates incubated for $24 \mathrm{hr}$.

The size of colonies in plate cultures was measured along two diameters, crossing at right angles, marked on the back of the plate and the average diameter of the colony calculated. To determine the growth rate this measurement was repeated at intervals of time. 
To determine the percentage germination of spores in liquid culture a sample was mounted in lactophenol cotton blue in a haemocytometer and the number of germinated spores in a total of 500 counted. A spore was considered to be germinated when the germ tube was as long as it was wide.

Nystatin solutions were prepared by dissolving commercial Squibb nystatin (3000 units/mg.) in propyleneglycol to give stock solutions of 12,000 units/ml. These were freshly prepared as required and all further dilutions were made in propyleneglycol. In all experiments, unless stated otherwise, $1 \mathrm{ml}$. nystatin solution was added to $19 \mathrm{ml}$. of molten GP agar cooled to $45^{\circ}$, or to $19 \mathrm{ml}$. of GP broth, to give final concentrations of $600,400,200,100,50,25,12 \cdot 50,6 \cdot 25,1 \cdot 60,0.80 \mathrm{units} / \mathrm{ml}$. An equal volume of solvent was added to the controls.

The sensitivity to nystatin of the Aspergillus species used in the present work was measured by a method (standard sensitivity test) described briefly here but in more detail elsewhere (English \& Stanley, in preparation). Molten GP agar incorporating nystatin at different concentrations, prepared as described above, was inoculated with $0.1 \mathrm{ml}$. of a standard spore suspension, mixed, and plates poured. The highest nystatin concentration at which microscopic examination showed a few spores to have germinated after incubation for $48 \mathrm{hr}$ was taken as the minimal inhibitory concentration (MIC; see Table 1).

Table 1. The minimal inhibitory concentration (MIC) of nystatin for four Aspergillus species determined by the standard sensitivity test

Nystatin ( $1 \mathrm{ml}$.) solution at a range of concentrations together with $0.1 \mathrm{ml}$. of a standard spore suspension were added to $19 \mathrm{ml}$. molten cooled GP agar, well mixed and plates poured. The lowest concentration inhibitory to germination was noted after incubation for $48 \mathrm{hr}$. at $37^{\circ}$.

MIC at $48 \mathrm{hr} . /($ units nystatin/ml.)

$\begin{array}{lc}\text { Aspergillus flavus } & \mathbf{1 2 \cdot 5} \\ \text { A. niger } & \mathbf{2 5} \\ \text { A. fumigatus } & \mathbf{2 5} \\ \text { A. fumigatus NyR } & \mathbf{5 0} \\ \text { A. terreus } & \mathbf{2 0 0}\end{array}$

\section{RESULTS}

The stability of nystatin

It is well known that nystatin is unstable under conditions likely to be used in experimental work (Kubitsa \& Derse, 1959; Eisenberg et al. 1956; Stewart, 1956). Since most of the experiments done in the present work were long-term growth studies it was necessary to know the rate of breakdown of nystatin during handling and under experimental conditions. Kinsky (1961) stated that the re-growth of the mycelium of Neurospora crassa, after $4 \mathrm{hr}$ at $25^{\circ}$ in the presence of the minimal inhibitory concentration of nystatin in liquid medium was due to photo-oxidation of the antibiotic. To determine the importance of the effect of light, flasks containing GP broth and plates of GP agar, both incorporating from 0 to 25 units nystatin $/ \mathrm{ml}$. were prepared. One series of each was stored for $24 \mathrm{hr}$ at room temperature in daylight and one in the dark. All series were then inoculated with a suspension of Saccharomyces cerevisiae and incubated for a further $24 \mathrm{hr}$ in the dark. In liquid culture the apparent amount of nystatin necessary to inhibit growth after 
storage in the light was double that necessary after storage in the dark. On the solid medium no difference was noted between plates stored in the light and in the dark. However, in all subsequent work nystatin solutions and plates were protected from light during manipulation, and incubation was always in the dark.

To determine the rate of breakdown of nystatin in the dark, series of GP agar plates containing from 0 to 600 units $/ \mathrm{ml}$. were prepared in triplicate. For each Aspergillus species one plate at each concentration was inoculated with $0.05 \mathrm{ml}$. of a standard spore suspension spread over a circular area $2.5 \mathrm{~cm}$. in diameter previously marked on the agar. The second and third series of plates were similarly inoculated after incubation for 24 and $48 \mathrm{hr}$ respectively. Further inoculations were made on each plate 24 and $48 \mathrm{hr}$ after the first. The minimal inhibitory concentrations for each series of inocula were noted at daily intervals. The results, which are the average of three experiments, are shown in Fig. 1. Other similar experiments were done with one series of plates only, the results of which confirmed those presented above. Therefore, using the minimal inhibitory concentration as a measure of the amount of nystatin remaining in the medium after incubation, it was apparent that the potency of the antibiotic was halved every $24 \mathrm{hr}$.

At nystatin concentrations which allowed growth during the first $48 \mathrm{hr}$, and after equal periods of incubation, the same amount of growth was obtained from inocula on plates on which the fungus was already growing as on those on which it was not. This indicates that loss in the potency of the nystatin was independent of the growth of the fungi and was due to spontaneous decomposition. In contrast to our results, Kubitsa \& Derse (1959) showed that there was a greater loss of potency of nystatin in plates on which a previous inoculum of Candida albicans had grown than on uninoculated plates. It is possible that in the present investigation the inocula were insufficiently close to one another for their zones of influence to interact, but since the distance between the edges of the original and subsequent inocula was only $c .1 \mathrm{~cm}$. any metabolic action of the Aspergillus species on nystatin must be slight.

At nystatin concentrations which prevented germination of Aspergillus spores during the first $48 \mathrm{hr}$ (i.e. $200 \mathrm{units} / \mathrm{ml}$. for Aspergillus terreus and 50 units $/ \mathrm{ml}$. for A. niger, A. fumigatus, A. flavus) a different effect was observed. The first inoculum on a fresh plate showed much less growth after a longer incubation period than later inocula on the same plate. The first inoculum would receive the full dose of nystatin, but even when this had deteriorated to a subinhibitory concentration further time was presumably required for the recovery of the majority of the spores or for the outgrowth of a few survivors. To confirm these results experiments were made using the length of the lag phase of growth (see next section) as a measure of the nystatin remaining in the medium. Plates of GP agar incorporating 0-200 units nystatin/ml. were prepared in triplicate. After incubation for $0,24,48 \mathrm{hr}$ one plate at each concentration was inoculated with $A$. fumigatus in the form of a seeded agar disc. The size of each colony was measured at intervals of time and the growth rate and length of the lag period before normal growth began were determined from the appropriate graphs. The results are shown in Fig. 2.

The length of the lag phase at each nystatin concentration decreased with increasing age of the plates and after the lag was overcome the final growth rates 
all reached those of the controls. At the lower concentrations the amount of antibiotic remaining after $24 \mathrm{hr}$ had fallen to a value which had no effect on the lag phase. Examination shows that the curves through each set of points are identical in shape and differ only in displacement along the log concentration axis; that is, the sole factor which affected the growth of the fungus was the loss in potency of the antibiotic with storage time. Read from the graph, the displacements of the curves for 24 and $48 \mathrm{hr}$ storage, respectively, are approximately log. $0 \cdot 4$ and log. $0 \cdot 5$, indicating that the potency of the nystatin was about halved every $24 \mathrm{hr}$.

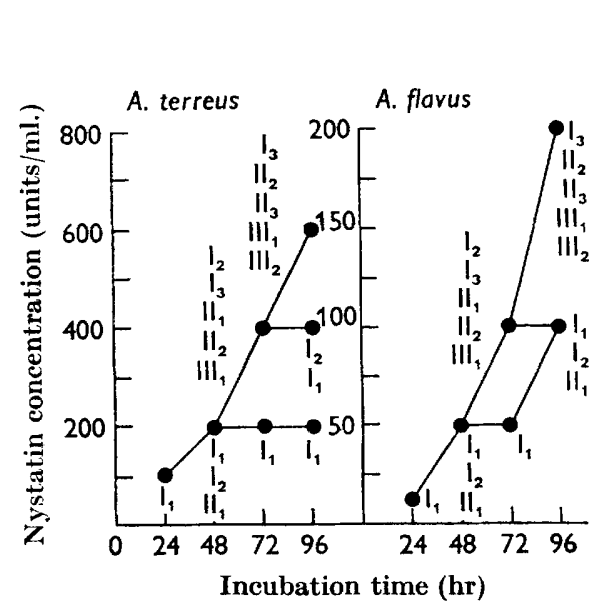

Fig. 1

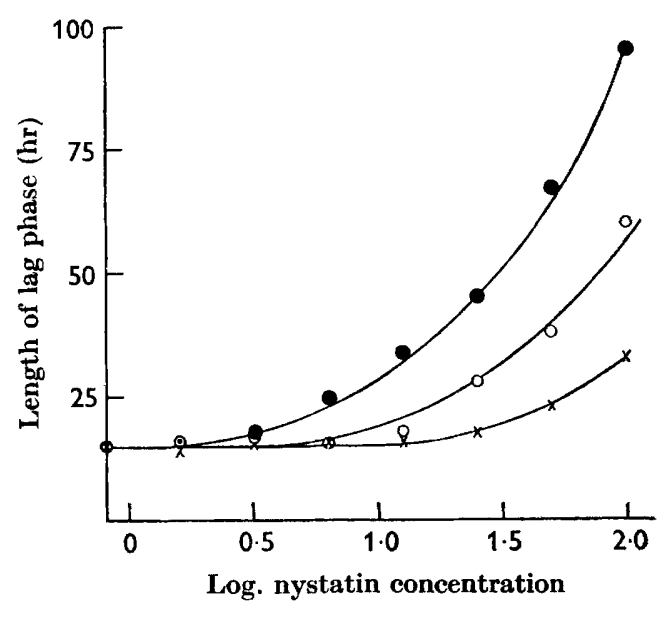

Fig. 2

Fig. 1. The change in the minimum concentration of nystatin necessary to inhibit Aspergillus terreus and $\boldsymbol{A}$. flavus caused by ageing the antibiotic for varying periods at $37^{\circ}$. Plates of GP agar incorporating from 0 to 600 units nystatin $/ \mathrm{ml}$. were prepared in triplicate and a plate at each concentration inoculated after the interval indicated with $0.05 \mathrm{ml}$. of a standard spore suspension spread over a circular area $2.5 \mathrm{~cm}$. diameter. The MIC for each series of inocula was noted at $24 \mathrm{hr}$ intervals. Time of inoculation after ageing plates at $37^{\circ}$ : Ist series of plates: $\left(I_{1}\right), 0 \mathrm{hr} ;\left(I_{2}\right), 24 \mathrm{hr} ;\left(I_{3}\right), 48 \mathrm{hr}$. 2nd series of plates: (II), $24 \mathrm{hr} ;\left(\mathrm{II}_{2}\right), 48 \mathrm{hr}$; $\left(\mathrm{II}_{3}\right), 72 \mathrm{hr}$. 3rd series of plates: $\left(\mathrm{III}_{1}\right), 48 \mathrm{hr}$; $\left(\mathrm{III}_{2}\right), 72 \mathrm{hr}$.

Fig. 2. The breakdown of nystatin, incorporated into GP agar at $37^{\circ}$, as demonstrated by the lag in growth rate of Aspergillus fumigatus. Plates of GP agar incorporating from 0 to 200 units nystatin $/ \mathrm{ml}$. were prepared, and plates at each concentration inoculated with a seeded agar disc $(0.9 \mathrm{~cm}$. diameter) at the times indicated. The diameters of the colonies were measured at intervals of time and the length of the lag phase determined from the growth curves. Time of inoculation after ageing plates at $37^{\circ}:(0), 0 \mathrm{hr} ;(O)$, $24 \mathrm{hr} ;(x), 48 \mathrm{hr}$.

The effect of nystatin on the length of the lag phase

The nature of the initial delay in growth caused by subinhibitory nystatin concentrations was investigated. For each Aspergillus species two series of GP agar plates were prepared, incorporating from 0 to 600 units nystatin/ml. Agar discs containing ungerminated spores were placed on one series of plates and similar discs containing mycelium on the other. The growth rate from each disc was measured. From the growth curves at each nystatin concentration the length of the lag phase was determined; the results are plotted in Figs. 3 and 4 . In each case, the final growth rates were the same as those of the controls (Fig. 3). Above a certain 
threshold value peculiar to each species the length of the lag increased to values which could not be explained solely by the increase in nystatin concentration. Figure 4 shows, for Aspergillus terreus at low nystatin concentrations, that the lag phase is longer for spores than for mycelium. This was also true of the other species tested, and is probably explained by the fact that spores neither swell nor germinate at inhibitory concentrations so that, after the nystatin has broken down to subinhibitory levels, additional time is necessary for this to occur before growth can begin. It also appears from Fig. 4 that the relationship between the length of the lag phase and the log. concentration of nystatin, while usually a smooth curve, may approach linear. Duplicate experiments showed that this occurred most often with $A$. flavus and $A$. niger: Lampen et al. (1959) noticed a similar phenomenon.

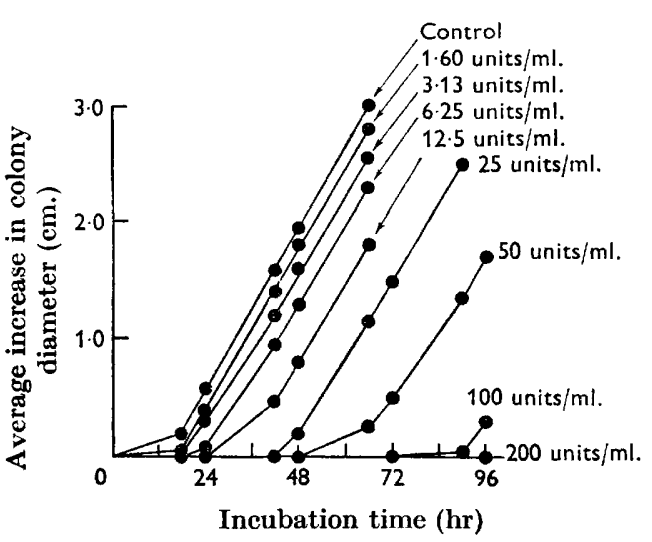

Fig. 3

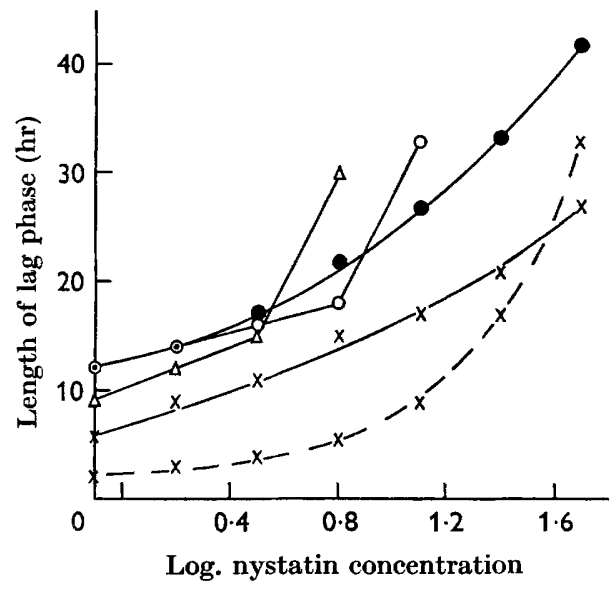

Fig. 4

Fig. 3. Effect of nystatin (units/ml.) incorporated into GP agar at concentrations indicated, on the radial growth of Aspergillus fumigatus. Plates of GP agar incorporating from 0 to 600 units nystatin $/ \mathrm{ml}$. were inoculated with dises seeded with spores or mycelium of Aspergillus fumigatus and the diameter of the colonies measured at intervals of time.

Fig. 4. Effect of nystatin incorporated into GP agar on the lag phase in growth of Aspergillus species. The length in lag phase in growth was determined from growth curves obtained as described in Fig. 3. Inoculum spores: $(\times), A$. terreus; $(O)$, A. niger $(\triangle), A$. flavus; (O), A. fumigatus. Inoculum mycelium: $(\times)$, broken line, $A$. terreus.

Since from this experiment it appears that the main effect of nystatin at subinhibitory concentrations was to lengthen the lag phase, further tests were made in an attempt to determine whether the ending of the lag was entirely due to the breakdown of nystatin or partly to adaptation by the fungi.

Test tubes of GP broth incorporating from 0 to 6000 units nystatin/ml. were inoculated with $1 \mathrm{ml}$. of a standard spore suspension of Aspergillus fumigatus to a total vloume of $6 \mathrm{ml}$. and incubated on a reciprocating shaker. After exposure for $0,5,15,30 \mathrm{~min}$. and 1, 4, 24 $\mathrm{hr}$ to nystatin, $1 \mathrm{ml}$. samples were taken, washed three times with large volumes of sterile distilled water to remove unabsorbed nystatin, and resuspended in $\mathbf{0 . 5} \mathrm{ml}$. water. One loop of this suspension was spread over a circular area $(0.9 \mathrm{~cm}$. diameter) on GP agar plates, the growth rate of the colonies measured, and the length of the lag phase calculated from the growth curves. After 
the lag phase, growth rates all reached that of the control. Exposure to nystatin, at all concentrations tested for periods up to $1 \mathrm{hr}$ resulted in only small increases in the lag period. After exposure for $4 \mathrm{hr}$ the lag period increased by a significant amount at all concentrations and the increase was greater at the higher concentrations. The spores of $A$. fumigatus at the concentration used were killed by 6000 units nystatin $/ \mathrm{ml}$. but not until they had been exposed for longer than $4 \mathrm{hr}$. Since in yeasts the inhibition of glycolysis caused by low concentrations of nystatin was annulled by $\mathrm{K}^{+}$(Cirillo, Harsch \& Lampen, 1964) the above experiment was repeated using $1 \%(\mathrm{w} / \mathrm{v}) \mathrm{KCl}$ for the washing and resuspension of the spores. Comparison with controls washed in water shows that $\mathrm{K}^{+}$had no significant effect on the length of the lag phase.

Table 2. Length of lag period of growth of Aspergillus fumigatus exposed to nystatin at $37^{\circ}$ after removal of unabsorbed antibiotic by washing in water

Time of exposure to nystatin

\begin{tabular}{|c|c|c|c|c|c|c|c|}
\hline $\begin{array}{l}\text { Nystatin } \\
\text { in GP broth }\end{array}$ & $1 \mathrm{~min}$. & 5 min. & $\begin{array}{l}15 \text { min. } \\
\text { Lag }\end{array}$ & $\begin{array}{l}30 \text { min. } \\
\text { period }(h\end{array}$ & $1 \mathrm{hr}$ & $4 \mathrm{hr}$ & $24 \mathrm{hr}$ \\
\hline 0 & 13 & 13 & 14 & 13 & 13 & 12 & 9 \\
\hline 6.25 & 13 & 13 & 16 & 15 & 18 & 21 & 28 \\
\hline 12.5 & 14 & 15 & 15 & 16 & 17 & 23 & 35 \\
\hline 25 & 13 & 14 & 15 & 14 & 18 & 22 & 24 \\
\hline 50 & 13 & 13 & 15 & 15 & 18 & 21 & 27 \\
\hline 100 & 14 & 15 & 15 & 15 & 19 & 22 & 34 \\
\hline 200 & 14 & 15 & 15 & 16 & 19 & 23 & 30 \\
\hline 600 & 14 & 13 & 15 & 16 & 17 & 25 & 36 \\
\hline 1000 & 14 & 16 & 15 & 16 & 16 & 28 & 47 \\
\hline 6000 & 17 & 17 & 18 & 17 & 19 & 38 & $\dagger$ \\
\hline
\end{tabular}

* The length of the lag phase was calculated from growth curves obtained from spores treated as described in Fig. 5.

$\dagger$ Lethal.

\section{Spore germination in the presence of nystatin}

Three series of $50 \mathrm{ml}$. flasks containing GP broth incorporating 0-100 units nystatin $/ \mathrm{ml}$. were prepared in duplicate, inoculated with $0.1 \mathrm{ml}$. of standard spore suspensions of Aspergillus niger, $A$. fumigatus and $\boldsymbol{A}$. fumigatus $\mathrm{NyR}$, respectively, and incubated on a reciprocating shaker. Samples were taken at intervals and the percentage germination determined. The average diameter of the spores was also measured. Since mycelium formed both in control flasks and at low nystatin concentrations after $18 \mathrm{hr}$, the final count was made at $12 \mathrm{hr}$. The results are plotted in Fig. 6 . Nystatin delayed swelling and germination of the spores of each species. The numbers germinated at each time interval decreased with increasing nystatin concentration in a linear fashion, until a concentration was reached at which no swelling of the spores occurred (50 units/ml. for $A$. fumigatus and $A$. niger, 100 units $/ \mathrm{ml}$. for $A$. fumigatus NyR). After incubation for $36 \mathrm{hr}$ the numbers of spores germinating at subinhibitory concentrations of nystatin had increased, and a few had germinated at a concentration which had been inhibitory at $12 \mathrm{hr}$. This was probably due to the normal decomposition of the nystatin, but the small numbers of spores which germinated at high concentrations suggests a possible fungicidal effect. 


\section{Fungicidal activity}

At low concentrations nystatin is fungistatic to a wide variety of yeasts and moulds, but at higher concentrations it becomes fungicidal (Bradley \& Jones, 1960). To determine the point at which this occurred with the Aspergillus species used

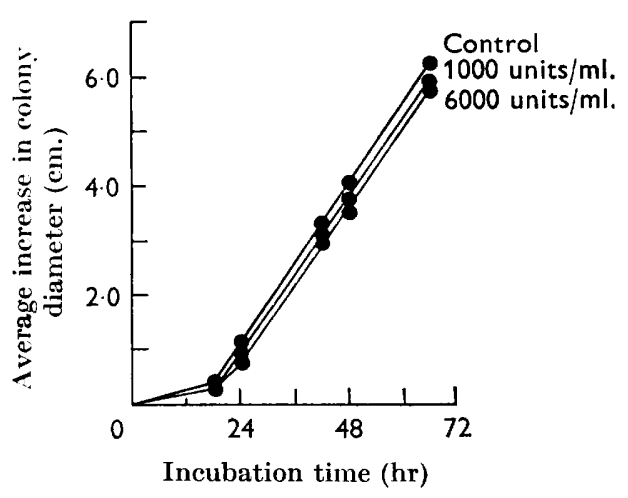

(a)

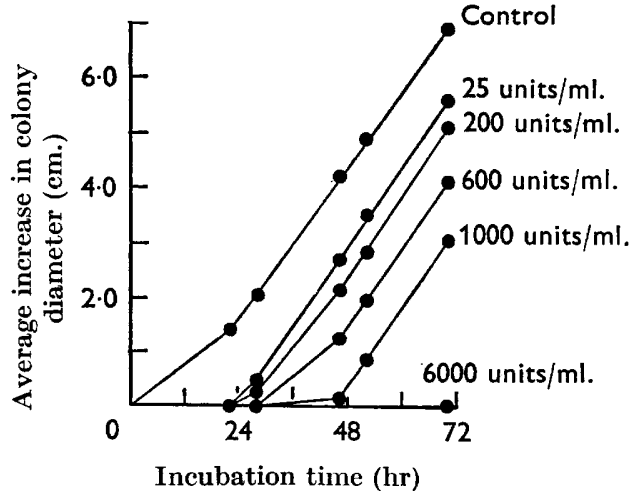

(b)

Fig. 5. Effect of time of exposure to nystatin (units $/ \mathrm{ml}$.) at the concentrations indicated on the growth rate of Aspergillus fumigatus. (a) Growth after exposure to nystatin for 5 min.; growth at intermediate concentration not shown as all values were very similar. (b) Growth after exposure to nystatin for $24 \mathrm{hr}$. $5 \mathrm{ml}$. of GP broth incorporating from 0 to 6000 units nystatin $/ \mathrm{ml}$. were inoculated with $1 \mathrm{ml}$. of a standard spore suspension of $A$. fumigatus (total vol. $6 \mathrm{ml}$.). $1 \mathrm{ml}$. samples were taken after $5,15,30 \mathrm{~min}$. and 1, 4, 24, $\mathrm{hr}$, the spores washed in water, resuspended in $0.5 \mathrm{ml}$. water, spread over a circular area of $0.9 \mathrm{~cm}$. diameter on GP agar and the diameters of the colonies measured at intervals of time.

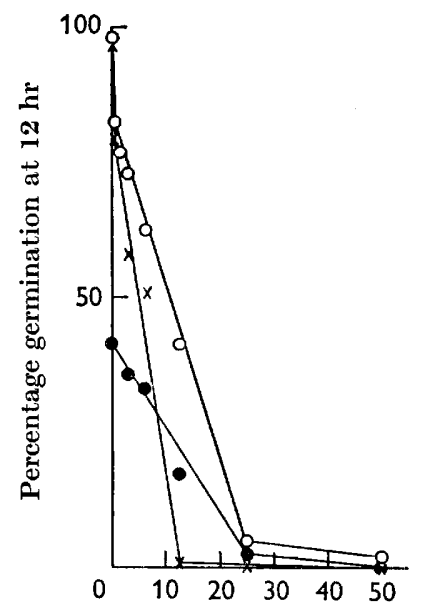

Nystatin concentration (units/ml.)

Fig. 6. Percentage germination of spores of Aspergillus species in the presence of nystatin incorporated into GP broth. GP broth incorporating from 0 to 100 units nystatin $/ \mathrm{ml}$. was inoculated with $0.1 \mathrm{ml}$. of a standard spore suspension (total vol. $20 \mathrm{ml}$.) and incubated on a reciprocating shaker. Samples were taken at intervals of time and the $\%$ germination determined by counting in a haemocytometer. (O), A. niger; (×), A. fumigatus; (O), A. fumigatus NyR. 
here, $1 \mathrm{ml}$. of a standard spore suspension of each Aspergillus species was inoculated into a series of test-tubes of GP broth +0 to 6000 units nystatin $/ \mathrm{ml}$. and incubated on a reciprocating shaker. Samples $(1 \mathrm{ml}$.) were taken after exposure for 1,4 , and $24 \mathrm{hr}$, washed as before and resuspended in $0.5 \mathrm{ml}$. sterile distilled water; $0.2 \mathrm{ml}$. samples of this suspension were spread over each of duplicate GP agar plates and the total number of colonies which developed within a week counted. The results are given in Table 3. No significant fungicidal action was noted after exposure for $1 \mathrm{hr}$ to any nystatin concentration used. After $4 \mathrm{hr}$ about $99.9 \%$ of the spores of Aspergillus flavus and $A$. niger were killed at all concentrations tested. The fungicidal action on $A$. fumigatus and $A$. terreus was less rapid. After $24 \mathrm{hr}$ at concentrations of over 100 units $/ \mathrm{ml}$. no spores of $A$. flavus or $A$. niger survived, but a few colonies of $A$. fumigatus developed after exposure to 1000 units $/ \mathrm{ml}$. and of $A$. terreus to 6000 units/ml. Lampen, Arnow \& Safferman (1960) have shown that even when $99.9 \%$ of yeast cells are killed, the remainder can grow when the unabsorbed nystatin is removed. Heavy inocula are known to be far less sensitive to fungicides than light inocula (Horsefall, 1954); in the present experiment clumping of the spores in shaken liquid culture might produce small foci of very heavy inocula which could explain the survival of a small number of spores for longer periods than the majority. It is clear, however, that the main fungicidal effect occurred at concentrations of nystatin only a few times higher than the fungistatic concentration.

Table 3. Death of Aspergillus spores caused by exposure to nystatin in glucose + peptone broth at $37^{\circ}$ for different times, followed by removal of the unabsorbed antibiotic by washing with water.

\begin{tabular}{|c|c|c|c|c|c|c|c|}
\hline \multirow{2}{*}{ Organism } & \multirow{2}{*}{$\begin{array}{c}\text { Time } \\
\text { of } \\
\text { exposure } \\
\text { (hr) }\end{array}$} & \multicolumn{6}{|c|}{ Concentration of nystatin (units/ml.) } \\
\hline & & $\mathbf{0}$ & 100 & $\begin{array}{l}200 \\
\text { No. }\end{array}$ & $\begin{array}{c}500 \\
\text { lonies }\end{array}$ & 1000 & 6000 \\
\hline \multirow[t]{3}{*}{ Aspergillus flavus } & 1 & $\infty$ & $\infty$ & $\infty$ & $\infty$ & $\infty$ & $\infty$ \\
\hline & 4 & $\infty$ & $60^{*}$ & $42 *$ & $40^{*}$ & 24* & $18^{*}$ \\
\hline & $\mathbf{2 4}$ & $\infty$ & $8^{*}$ & 0 & $\mathbf{0}$ & $\mathbf{0}$ & $\mathbf{0}$ \\
\hline \multirow[t]{3}{*}{ A. niger } & 1 & $\infty$ & $\infty$ & $\infty$ & $\infty$ & $\infty$ & $\infty$ \\
\hline & 4 & $\infty$ & $167 *$ & $112 *$ & $89^{*}$ & $92 *$ & $75 *$ \\
\hline & 24 & $\infty$ & $\mathbf{5}^{*}$ & 0 & $\mathbf{0}$ & 0 & 0 \\
\hline \multirow[t]{3}{*}{ A. fumigatus } & 1 & $\infty$ & $\infty$ & $\infty$ & $\infty$ & $\infty$ & $\infty$ \\
\hline & 4 & $\infty$ & $\infty$ & $\infty$ & $\infty$ & $\infty$ & $\infty$ \\
\hline & 24 & $\infty$ & $31 *$ & $9^{*}$ & $16^{*}$ & $19^{*}$ & 0 \\
\hline \multirow[t]{3}{*}{ A. terreus } & 1 & $\infty$ & $\infty$ & $\infty$ & $\infty$ & $\infty$ & $\infty$ \\
\hline & 4 & $\infty$ & $\infty$ & $\infty$ & $\infty$ & $\infty$ & $\infty$ \\
\hline & 24 & $\infty$ & $155^{*}$ & $112 *$ & $122 *$ & $78 *$ & $98^{*}$ \\
\hline
\end{tabular}

The effect of more prolonged exposure to moderate concentrations of nystatin was investigated for three growth phases. Standard suspensions of ungerminated and germinated spores of each Aspergillus species were streaked on GP agar + 600 units nystatin/ml. After incubation for $48 \mathrm{hr}$ the inocula were scraped off and plated on fresh GP agar. The ungerminated spores of all species and the germinated spores of $A$. terreus all gave numerous colonies although growth was delayed from 1 to 2 days; but in comparison with the controls the colonies were less numerous, suggesting 
some killing. All the germinated spores of $A$. niger were killed, but a few colonies developed from those of $A$. flavus and $A$. fumigatus after 3-5 days. This might have been due to the presence of a few ungerminated spores in the inoculum or to clumping. When forty mycelial balls of each species were exposed to 600 units nystatin $/ \mathrm{ml}$. in GP broth (total volume $6 \mathrm{ml}$.) for $48 \mathrm{hr}$, then washed and replated on GP agar, growth occurred only from the mycelium of $A$. terreus.

\section{Microscopical changes in Aspergillus germ tubes after exposure to nystatin at inhibitory concentrations}

To obtain Aspergillus spores at different stages of development, pieces of cellulose film laid over GP agar were inoculated with spores of each Aspergillus species and incubated. Pieces of film were removed at intervals and placed on the surface of GP agar +100 units nystatin $/ \mathrm{ml}$. for Aspergillus niger, $A$. flavus and $A$. fumigatus and $400 \mathrm{units} / \mathrm{ml}$. for $A$. terreus. After incubation for $24 \mathrm{hr}$ the cellulose strips were mounted in lactophenol cotton blue and examined microscopically. In each case growth was inhibited at the stage reached before contact with nystatin. The germ tubes of $A$. niger and particularly of $A$. flavus were greatly plasmolysed in comparison with refrigerated controls. The germ tubes of $\boldsymbol{A}$. fumigatus contained numerous refractile granules not seen in the controls. No differences were observed in A. terreus.

\section{Induction of resistance to nystatin}

The possibility that the growth shown by the Aspergillus species at high nystatin concentrations after the long lag phase was due to induced resistance to nystatin was examined. Gradient plates (Szybalski, 1952) at a maximum strength of 600 units nystatin $/ \mathrm{ml}$. were prepared; $0.1 \mathrm{ml}$. of a spore suspension of each Aspergillus species was spread over the plates which were then incubated for $72 \mathrm{hr}$. Colonies which developed at the highest nystatin concentration were picked off and subcultured on further gradient plates at the same concentration of nystatin, in the manner just described. After ten and twenty transfers the sensitivity of the isolates was determined by the standard sensitivity test. There was no increase of resistance in Aspergillus niger or A. flavus, but a strain of $A$. fumigatus with double the resistance of the parent strain was obtained, i.e. with the same resistance as $A$. fumigatus NyR. No further increase of resistance was observed in $A$. fumigatus NyR. As a further check on the possible occurrence of adaptation or the selection of resistant strains, spores which survived exposure to high concentrations of nystatin in some of the experiments previously described were tested by the standard sensitivity test, but no increase in resistance was found.

\section{DISCUSSION}

Direct experiments on the sensitivity of the fungi suggested that, within each Aspergillus species, the level of nystatin necessary to inhibit spore germination was the same as that needed to inhibit hyphal growth; but experiments on the lag phase showed that mycelium was more sensitive than spores, and the lethal concentration of the drug was also shown to be lower for mycelium than for spores. The probable explanation of this inconsistency is that the doubling increments of nystatin used throughout this work were too large to detect small differences in the 
minimal inhibitory concentration. This could also account for our finding no difference in the minimal inhibitory concentration in liquid and in solid media, contrary to the results of Stewart (1956) who, working with yeasts, found that more nystatin was required to inhibit growth in solid than in liquid media. An indication in our experiments that this is, in fact, true for Aspergillus species was that, as nystatin deteriorated with time, more spores germinated in initially inhibitory solid media than in liquid media containing the same amount of nystatin. Better contact between spore and antibiotic in shaken, liquid cultures than in solid ones is the probable explanation.

Marini, Arnow \& Lampen (1961) have demonstrated that in Saccharomyces cerevisiae, even if the inhibition of glycolysis which is caused by nystatin is reversed by the addition of $\mathrm{K}^{+}$etc., there may still be a complete loss of viability of some cells: these workers showed that this irreversible change was due to the additional loss of cytoplasmic constituents other than those necessary to maintain glycolysis. This probably also explains our finding of the marked lengthening in the lag phase of the Aspergillus species at concentrations approaching the inhibitory level, and the high proportion of spores killed by nystatin at concentrations only a few times greater than those causing fungistasis.

The necessity for a minimum length of exposure of Aspergillus spores to nystatin before any effect at all can be observed, even at the highest antibiotic concentrations, is consistent with the findings of Lampen, Arnow, Borowska \& Laskin (1962) that in yeasts the intact cell-wall delays binding and that the binding sites do not appear to be readily accessible. Nystatin absorbed on to the organism is not removed by washing with buffer or acetone, or by adjusting the $\mathrm{pH}$ (Lampen et al. 1959), and in yeasts the rate of absorption and total amount absorbed increases as the nystatin concentration is raised (Lampen \& Arnow, 1959). This suggests that the lengthening of the lag phase of Aspergillus species is also due to increased absorption of nystatin, this phase eventually being terminated when the absorbed nystatin breaks down to a subinhibitory level.

Differences in the rate at which nystatin is absorbed at the cell surface, combined with the decay of the absorbed antibiotic with time, might explain differences in the behaviour of the individual Aspergillus species in the presence of the antibiotic. In the standard sensitivity test, where incubation was for a period of $48 \mathrm{hr}$ in continued contact with nystatin, maximum absorption must occur and germination be inhibited at a relatively low external concentration. In the fungicidal tests, on the other hand, unabsorped nystatin was removed after limited exposure periods, and if absorption were slow in some species, recovery of survivors, even after exposure to very high concentrations, might be expected. This would appear to be the case with Aspergillus terreus, but cannot alone explain the comparative resistance of $A$. fumigatus to the fungicidal action of nystatin, when coupled with its extreme sensitivity to fungistasis.

Strains of filamentous fungi or yeasts which have developed resistance to nystatin have rarely been reported in the literature and the general opinion is that resistance of the type occurring with other antibiotics is not met with (Stout \& Pagano, 1956). Where resistance does occur, as in Aspergillus fumigatus (Manning \& Robertson, 1959), Candida albicans, Cerastomella ulmi (Stout \& Pagano, 1956) and Candida spp. (Littman, Pisano \& Lancaster, 1957-58), it is only two or three times that of 
the original strain and it does not appear possible to increase it further. These observations have been confirmed with the four Aspergillus species used in this investigation.

Since it has been shown that nystatin undergoes natural degradation at $37^{\circ}$, that there is survival of a small number of spores at high nystatin concentrations, and that these survivors have the normal growth rate and no increased resistance, it would appear that the growth which follows exposure to nystatin at high concentrations for long periods of time is not due to adaptation, or to the selection of resistant spores, but to the slow outgrowth of a few normal survivors as the nystatin loses potency.

We are indebted to the Medical Research Council for the grant which supported this work. We wish to thank Dr A. H. Linton of the University of Bristol for most valuable advice and criticism, and the Dispensary of the Bristol General Hospital for preparation of the nystatin stock solutions.

\section{REFERENCES}

Bradley, S. G. \& Jones, L. A. (1960). Mechanisms of action of antibiotics. Ann. N. Y: Acad. Sci. 89, 122.

Cirillo, V. P., Harsch, M. \& Lampen, J. O. (1964). Action of the polyene antibiotics Filipin, nystatin and $\mathrm{N}$-acetylcandidin on the yeast cell membrane. J. gen. Microbiol. 35, 249.

Eisenberg, G. M., Weiss, W. \& Fuippin, H. F. (1956). Microbial studies and preliminary clinical experiences with nystatin. Antibiot. Ann., 1955-56, p. 837.

Horsefald, J. G. (1945). Fungicides and their action. Annales Cryptogamici et Phytopathologici, vol. 2. Ed. by F. Verdoon. Waltham, Mass. U.S.A.: Chronica Botanica Co.

KINSKY, S. C. (1961). Alterations in the permeability of Neurospora crassa due to polyene antibiotics. J. Bact. 82, 889.

KINSKY, S. C. (1962). Effect of polyene antibiotics on protoplasts of Neurospora crassa. J. Bact. 83, 351.

Kubitsa, R. A. \& Derse, P. H. (1959). A comparison of triacetin and nystatin with respect to their stability and effect on Candida albicans. Antibiot. \& Chemother. 9, 546.

Lampen, J. O. \& Arnow, P. (1959). Significance of nystatin uptake for its antifungal action. Proc. Soc. exp. Biol. Med. 101, 792.

LAMPEN, J. O. \& ARNow, P. (1961). Inhibition of algae by nystatin. J. Bact. 82, 247.

Lampen, J. O., Arnow, P. M. \& Safferman, R. S. (1960). Mechanism of protection by sterols against polyene antibiotics. J. Bact. 80, 200.

Lampen, J. O., Arnow, P. M., Borowska, Z. \& Laskin, A. I. (1962). Location and role of sterol at nystatin binding sites. J. Bact. 84, 1152.

Lampen, J. O., Morgan, E. R., Slocum, A. \& Arnow, P. (1959). Absorption of nystatin by microorganisms. J. Bact. 78, 282.

Litrman, M. L., Pisano, M. A. \& Lancaster, R. M. (1958). Induced resistance of Candida species to nystatin and amphotericin B. Antibiot. Ann. 1957-58, p. 981.

Manning, L. K. \& Robertson, B. M. (1959). A case of aspergillosis treated with nystatin. Br. med. J. i, 345

Marini, F., Arnow, P. M. \& Lampen, J. O. (1961). The effect of monovalent cations on the inhibition of yeast metabolism by nystatin. J. gen. Microbiol. 24, 51.

STEwart, G. T. (1956). Laboratory and clinical studies with nystatin in post-antibiotic mycotic infections. Br. med. $J$. i, 658.

Stout, H. A. \& Pagano, J. F. (1956). Resistance studies with nystatin. Antibiot. Ann. 1955-56, 704.

Szybalski, W. (1952). Microbial selection 1. Gradient plate technique for study of bacterial resistance. Science, 116, 46. 